\title{
Enhancing Serial Block-Face Scanning Electron Microscopy to Enable High Resolution 3-D Nanohistology of Cells and Tissues
}

\author{
T. J. Deerinck*, E. A.Bushong*, V. Lev-Ram**, X. Shu**, R.Y. Tsien** and M. H. \\ Ellisman*
}

*Center for Research in Biological Systems and the National Center for Microscopy and Imaging Research, **Howard Hughes Medical Institute and Dept. of Pharmacology, University of California, San Diego, La Jolla, CA, 92093

Serial block face scanning electron microscopy (SBFSEM) is a powerful technique originally introduced by Leighton [1], substantially improved by Denk [2] and subsequently commercialized (Gatan Inc., Pleasanton, CA.). SBFSEM allows for the automated image acquisition of relatively large volumes of tissue at near nanometer-scale resolution, using a dry cutting ultramicrotome fitted into an SEM. In an automated process, a low voltage backscatter electron (BSE) image is obtained from the surface of an epoxy embedded tissue block face. The ultramicrotome then removes an ultra-thin section of tissue with a specially designed oscillating diamond knife (Diatome AG, Switzerland), and a block face image from the corresponding region is again obtained. This sequence is repeated over and over until the desired volume of tissue has been imaged. Although SBFSEM overcomes many obstacles routinely encountered with serial section TEM reconstruction, until recently there was a significant limitation to the resolution obtainable by this method compared to conventional TEM. This was due primarily to difficulties encountered using BSE imaging at low accelerating voltages. To overcome this we have developed a protocol for vastly increasing the heavy metal staining of specimens to improve BSE yield. This is accomplished by combining a variety of preexisting heavy metal staining methodologies not normally used together, including ferrocyanide-reduced osmium tetroxide, thiocarbohydrazide-osmium tetroxide (OTO), prolonged uranyl acetate treatment and en bloc lead aspartate staining. Using this approach, we demonstrate a dramatic improvement in image contrast and resolution from existing methods in a variety of specimens (Fig. 1).

We have also combined this approach with a number of selective labeling methods such as Golgi impregnation to allow the reconstruction of whole cells in the nervous system (Fig. 2), as well as fluorescence photoconversion to label specifically targeted proteins [3,4]. Additionally, a powerful application of SBFSEM is its use in conjunction with a newly developed genetically encoded fluorescent reporter termed miniSOG (for mini singlet oxygen generator). MiniSOG is a small (106residue) singlet oxygen generating protein engineered from a flavin-binding, blue light phototropin from Arabidopsis thaliana. MiniSOG has quantum yields for fluorescence and singlet oxygen of 0.30 and 0.47 respectively. It can be genetically fused to the target protein of interest for both fluorescence imaging and efficient photooxidation of diaminobenzidine into an osmiophilic polymer for subsequent electron microscopy [3]. Since miniSOG is genetically encoded and all other reactants $\left(\mathrm{O}_{2}\right.$, diaminobenzidine, $\left.\mathrm{OsO}_{4}\right)$ are permeant small molecules, there is no need to compromise chemical fixation to preserve protein epitopes or to permeabilize with detergents, which further degrade cellular ultrastructure. We have combined this approach with the intense heavy metal staining procedure outlined above for SBFSEM to enable 3D localization of genetically specified proteins in cells and tissues even from intact mice. This approach should enable a new era of automated 3D-nanohistology of a wide range of tissues and organisms. 
References

[1] S. Leighton, Scan Electron Microsc. (1981) (Pt 2):73-6.

[2] W. Denk and H. Horstmann. PLoS Biol. (2004) 2(11):e329.

[3] T. Deerinck M. Martone, V. Lev-Ram, D. Green, R. Tsien, D. Spector, S. Huang and M. Ellisman. J. Cell Biol. (1994) 126(4):901-10.

[4] G. Gaietta, T. Deerinck, S. Adams, J. Bouwer, O. Tour, D. Laird, G. Sosinsky, R. Tsien and M. Ellisman. Science. (2002) Apr 19; 296(5567):503-7.

[5] This work was supported by NIH grant RR-P41004050 to MHE and NIGMS grant R01 GM086197 to RYT.

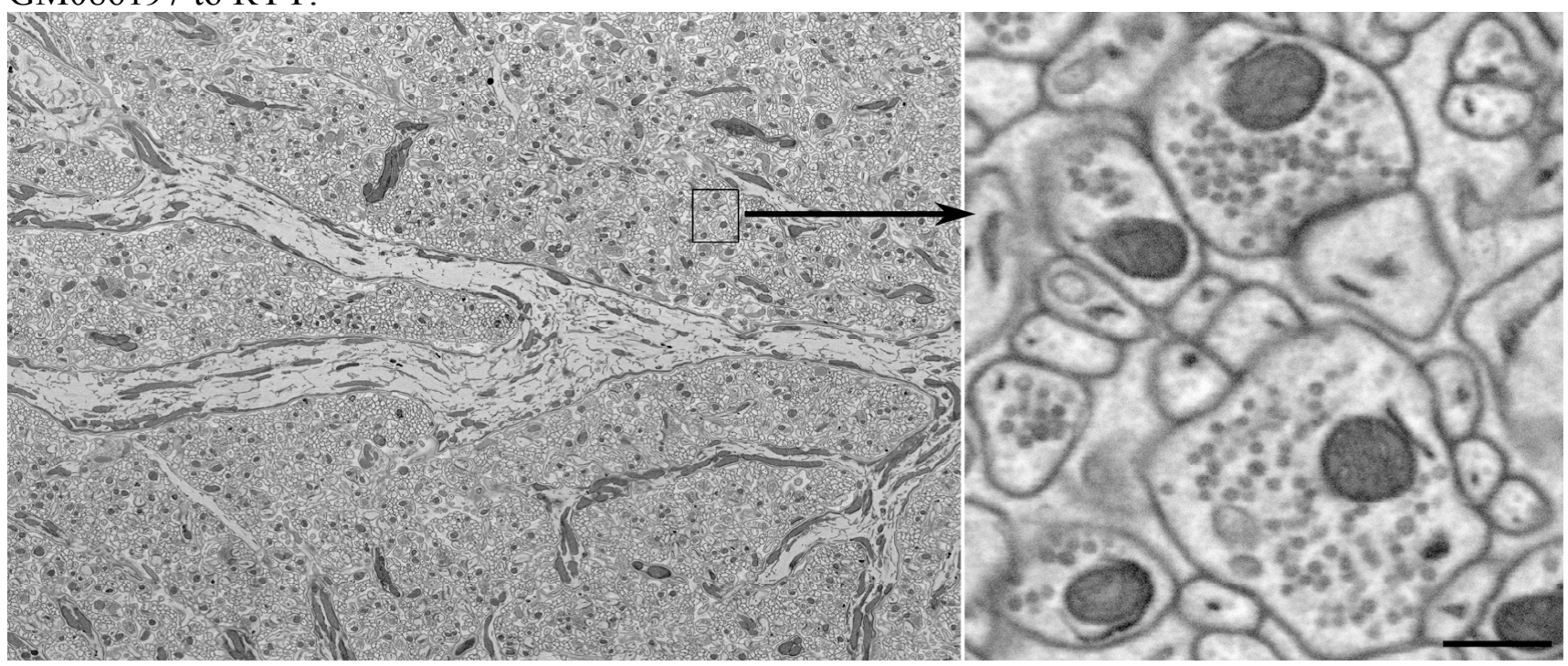

FIG. 1. A portion of a 16k x 24k pixel BSE block face image of mouse cerebellum (enlargement of boxed region shown on right) showing the excellent image resolution obtainable using the combined en bloc OTO-uranium-lead staining. Imaged at $2.0 \mathrm{keV}$ and at high vacuum. Bar $=250 \mathrm{~nm}$.

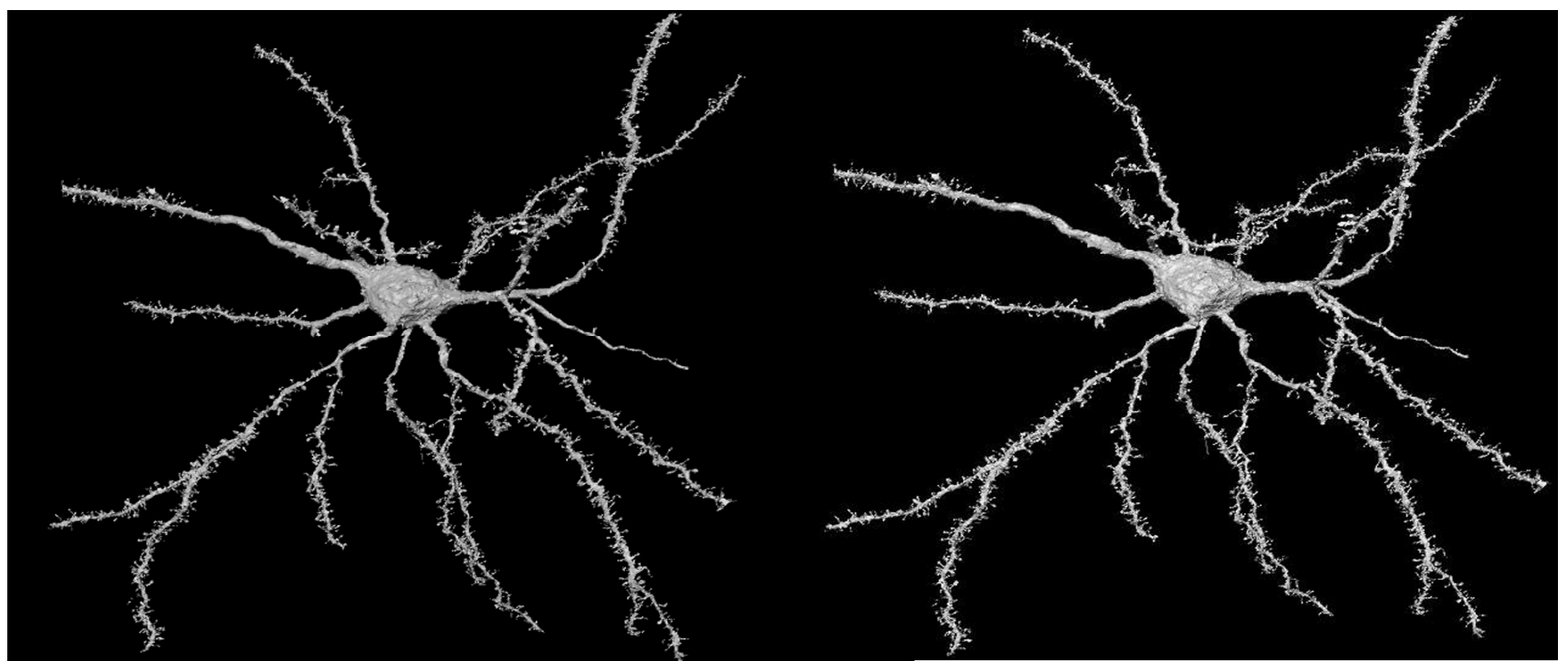

FIG. 2. Stereopair image (viewed cross-eyed) of a Golgi impregnated medium spiny cortical neuron reconstructed from a volume obtained by automated SBFSEM. Surface rendering of contrastreversed maximum intensity projection. Field of view: 100 microns. 